\title{
It's Rare So Be Aware: Pleuropulmonary Blastoma Mimicking Congenital Pulmonary Airway Malformation
}

\author{
Fayza Haider ${ }^{1}$ Khulood Al Saad ${ }^{2}$ Fatima Al-Hashimi ${ }^{3} \quad$ Hakima Al-Hashimi $^{4}$ \\ 1 Pediatric Surgery Unit, Department of Surgery, Salmaniya Medical \\ Address for correspondence Fayza Haider, MD, MRCSI, CABS, \\ Complex, Manama, Bahrain \\ 2 Pediatric Department, Salmaniya Medical Complex, Manama, \\ Pediatric Surgery Unit, Department of Surgery, Salmaniya Medica \\ Complex, P.O. Box 12, Manama, Bahrain \\ Bahrain \\ ${ }^{3}$ Department of Pathology, Salmaniya Medical Complex, Manama, \\ (e-mail: drfayzahaider@gmail.com; fali1@health.gov.bh). \\ Bahrain \\ ${ }^{4}$ Department of Radiology, Salmaniya Medical Complex, Manama, \\ Bahrain \\ Thorac Cardiovasc Surg Rep 2017;6:e10-e14.
}

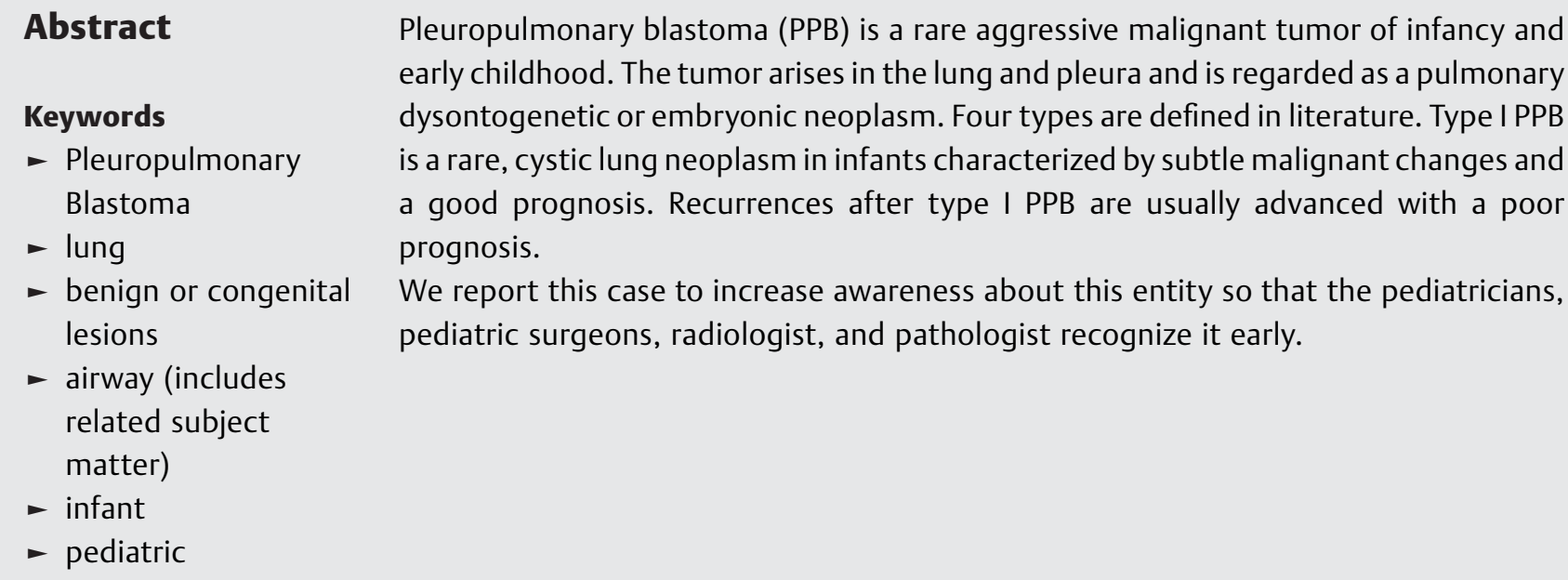

\section{Introduction}

Pleuropulmonary blastoma (PPB) is a rare, aggressive, dysontogenetic, and malignant tumor of intrathoracic (pulmonary, pleural, or combined) mesenchyme. ${ }^{1}$

It is the most common primary malignancy of the lung in childhood. It was initially proposed to be a distinct entity in $1988 .^{2}$ Three pathologic types in the evolution of PPB have been defined: type Ior purely cystic, type II or cystic/solid, and type III or purely solid. The progression of type I to types II and III is well documented.NotallcystictypeIPPBsare destined toprogress to the more malignant types. These "nonprogressed/regressed" cystic cases are designated as type I regressed (type Ir). ${ }^{3}$
In the newborn or older infant, purely cystic type I PPB is readily mistaken for one of several congenital cystic lesions of the lungs such as congenital pulmonary airway malformation (CPAM). Such lesions are more likely to be PPB if there is a family history of PPB or associated tumors, but ultimately a pathologic examination is required. ${ }^{4}$

CPAMs encompass a varied group of disorders that include congenital cystic adenomatoid malformation (CCAM), bronchopulmonary sequestration (BPS), congenital lobar emphysema, and bronchogenic cyst among other entities.

CPAM is relatively rare, with a reported incidence between 1:25,000 and 1:35,000. ${ }^{5}$ CCAM was first described by Ch'in and Tang in 1949, ${ }^{6}$ and is now also known as CPAM received

October 30, 2016

accepted

January 10, 2017
DOI http://dx.doi.org/

$10.1055 / \mathrm{s}-0037-1598625$.

ISSN 2194-7635.
(C) 2017 Georg Thieme Verlag KG Stuttgart · New York

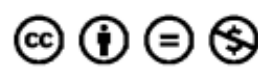




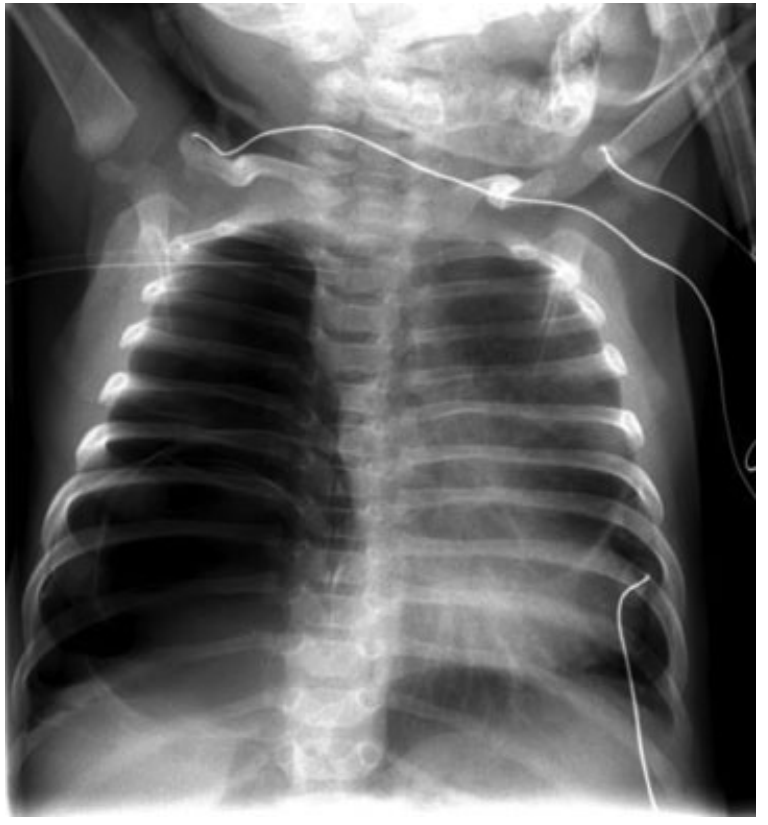

Fig. 1 AP chest radiograph.

due to the new classification system established by Stocker et al.

There are five types of CPAM including tracheobronchial (type 0 ), bronchial/bronchiolar (type 1 ), bronchiolar (type 2 ), bronchiolar/alveolar (type 3), and distal acinar (type 4), classified according to the location of the developmental site of malformation. ${ }^{7}$

Type I PPB and CCAM are cystic lung diseases occurring in neonates or in young children. Differentiating between them remains a challenge for pathologists, and type I PPB is currently confused with CCAM. $^{7}$

We are reporting our case to increase awareness about this entity so that the pediatricians, pediatric surgeons, radiologist, and pathologist recognize it early.

\section{Case Presentation}

Our case is a 3-month-old male baby, who was born by cesarean section for big size, as birth weight was $4.4 \mathrm{~kg}$ secondary to gestational diabetes. There were no reported anomalies in the antenatal ultrasound. Hehad no neonatal complication and was doing well until his presentation.

He had a 2-week history of cough associated with difficulty in breathing, for which the family sought medical advice and was given treatment as bronchial asthma. Because there was no improvement, he was taken to a pediatric pulmonologist who noticed significant reduced air entry on the right side with mild respiratory distress. Chest X-ray was done immediately, which showed a picture of emphysematous lesion versus a large multiloculated cystic mass in the hemithorax, displacing the heart and mediastinum to the left side indicating a possibility of pneumothorax; hence, an intercostal drain (ICD) was inserted for this high possibility (-Fig. 1). The baby was referred immediately for admission at our institute. An urgent CT of the chest was done, which showed a large multiloculated cystic mass measuring at its largest diameter $(9.2 \times 4.2 \times 3.3 \mathrm{~cm})$, occupying most of the right hemithorax. The cystic spaces are air-filled, causing significant mass effect, displacing the heart to the left side. Part of normal appearing right lung is seen anterior to the heart (-Fig. 2). CT angiogram (not included) from the same study does not show any systemic arterial supply to the mass, excluding sequestration.

On the 4th day of his admission, the parents consented for surgery as his condition was not improving. He underwent right thoracotomy and right lung lower lobectomy. Intraoperatively, a large multilobulated cystic lesion was found occupying the whole right hemithorax (-Figs. 3, 4). The rest of the lung was normal, and it expanded well after the resection of the right lower lobe.

The histopathology report came later with the diagnosis of PPB type 1. The normal lung parenchyma is replaced by cysts that are lined by focally hyperplastic columnar epithelium and the lung parenchyma is replaced by medium-sized blue cells (-Fig. 5). The pleura is also replaced by sheets of medium-sized blue cells, with immature elements. Differentiation of these cells was further classified on immunostains. There is a cambium zone of variable thickness composed of round to spindle-shaped immature mesenchymal cells interspersed with few bundles of smooth muscle. The epithelial cells are positive for AE1/3. The mesenchymal cells are positive for desmin and smooth muscle actin (SMA) (-Fig. 6).

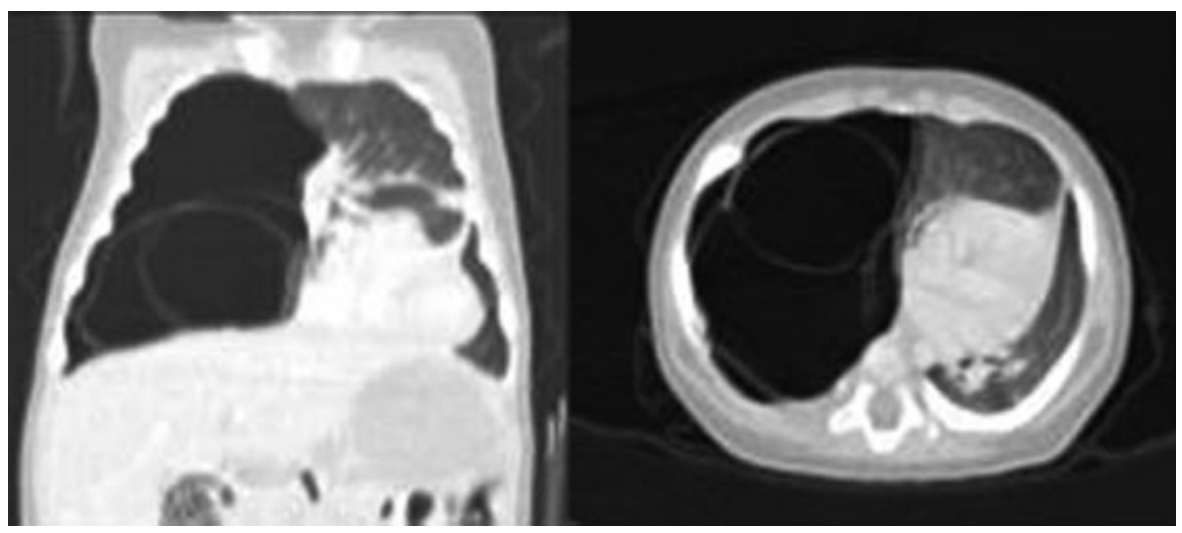

Fig. 2 Axial CT cuts of the chest. 


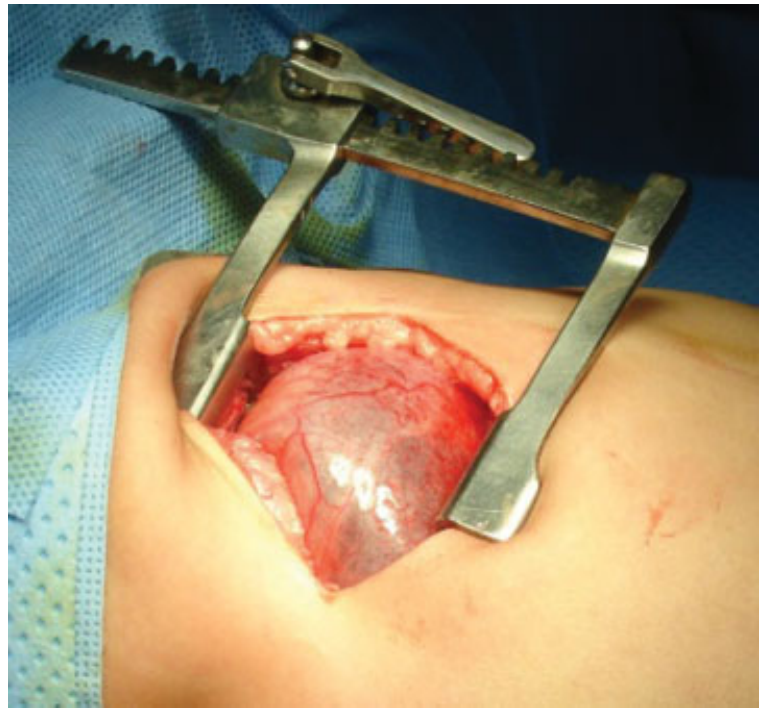

Fig. 3 Cystic lesion.

He was referred to the oncology clinic where a further history documented no family history of pulmonary tumors, lung or renal cysts, or brain tumors. This was confirmed by renal ultrasound and brain MRI.

The family agreed to register him in the International PPB Registry where the blocks were sent for confirmation. The case is registered as case 591, and the diagnosis was confirmed.

The parents were not willing to consider adjuvant chemotherapy at this stage unless there is evidence of relapse.

His follow-up CT at 3 months showed no evidence of residual or relapsed lung lesions. His 6 months follow-up CT showed a spiculated soft tissue nodule at the medial basal segment at operative resection site. Concern was raised about the possibility of recurrent tumor (-Fig. 7). Therefore,

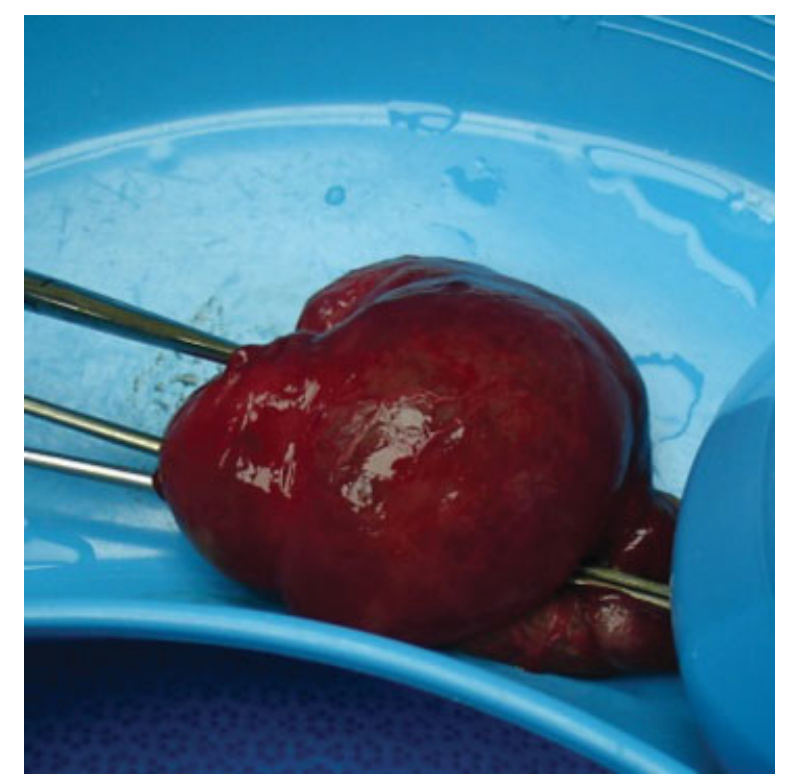

Fig. 4 Resected right lower lobe.

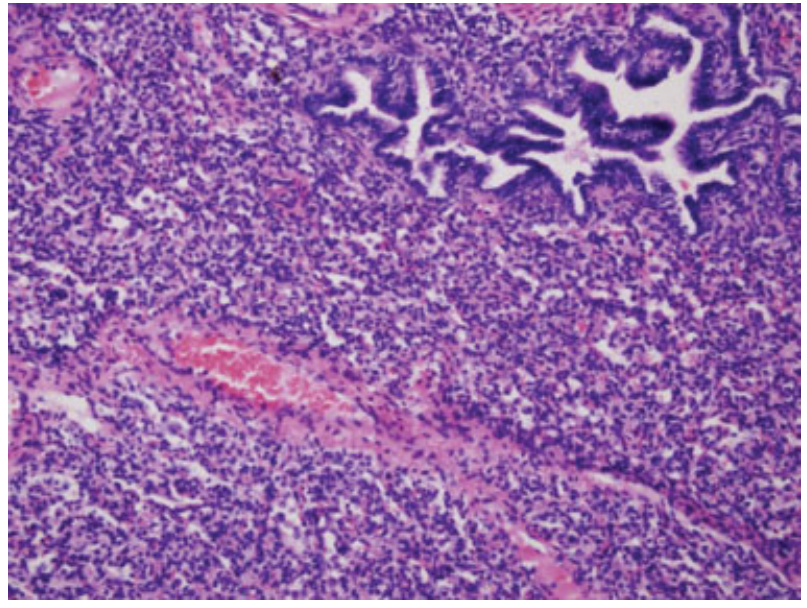

Fig. 5 H\&E: $\times 200$ The resected lung.

he was taken for a second look and the mentioned lesion was resected. The histopathology of the resected lesion came as nonspecific foci of emphysema and atelectasis only with no evidence of tumor.

Because of the suspicion of recurrence and for a better outcome with adjuvant chemotherapy for type I PPB, the parents finally agreed.

He is now 2 years in follow-up and is disease free and under surveillance (-Fig. 8).

\section{Discussion}

Pleuropulmonary blastoma is a rare intrathoracic tumor accounting for $15 \%$ of all primary pediatric pulmonary tumors. $^{8}$

There are several clinical and radiologic features that are predictive for $\mathrm{PPB}$, including symptoms (particularly pneumothorax), bilateral or multisegment involvement, the presence of a complex cyst, and a germline mutation in the DICER1 gene, found in two-thirds of PPB. Our patient had a positive germline mutation in the DICER1 gene, and the

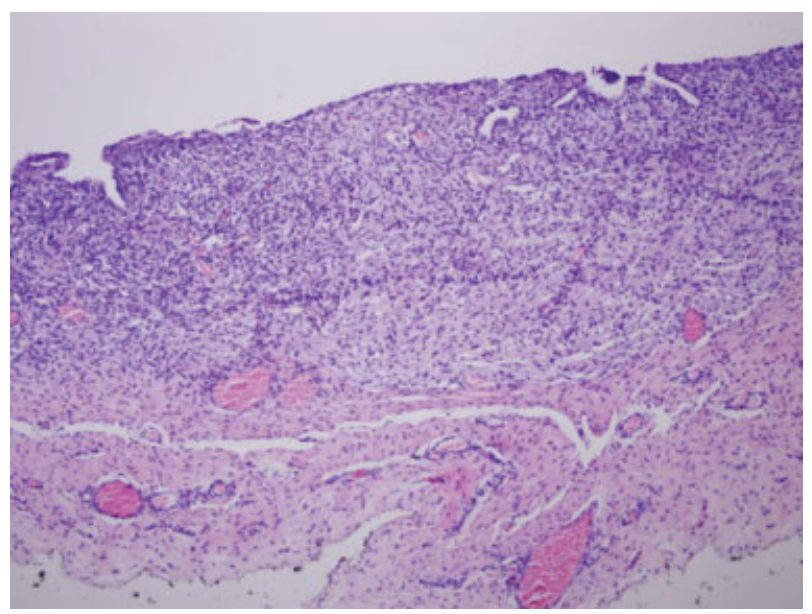

Fig. 6 H\&E: $\times 100$. The pleura. 


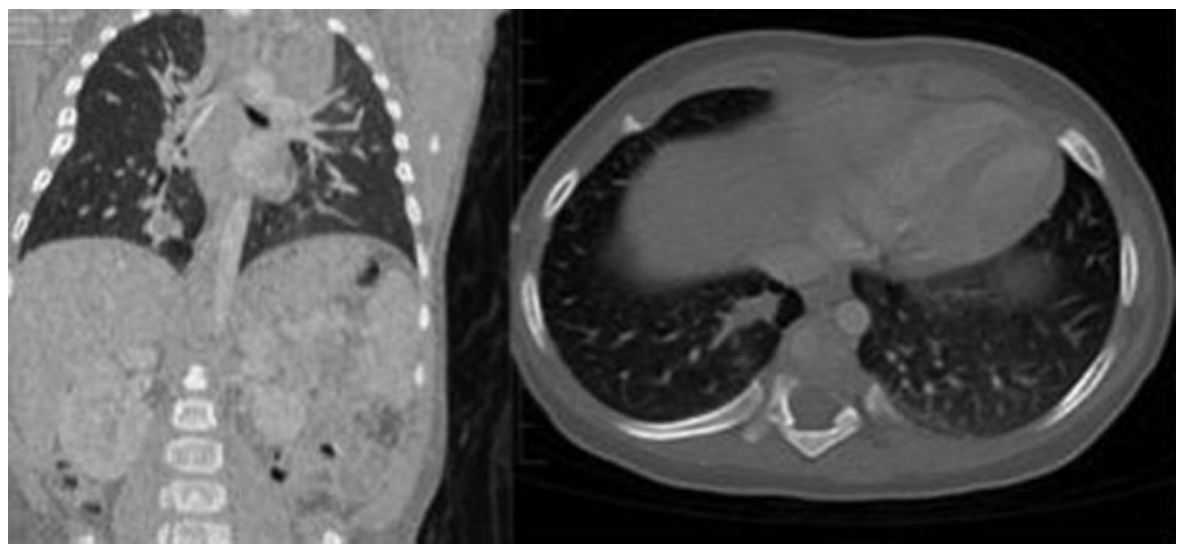

Fig. 7 Six-month follow-up CT.



Fig. 8 Two-year follow-up.

affected parent was the father. In contrast, the features that were predictive for CPAM included prenatal diagnosis, systemic feeding vessel, and hyperinflated lung. ${ }^{9}$

In the case reported, pneumothorax was highly suspected, but it was unilateral with involvement of a single segment with a definite complex cyst, so he had two out of four of the symptoms, as concluded by Feinberg et al.

Several studies have suggested that these two entities can be indistinguishable based on imaging, and there is overlap between type 4 CCAM and type I PPB on pathology. ${ }^{10}$

It may be difficult for even experienced pathologists to correctly identify a lesion as PPB, so a central pathology review has helped clarify questionable cases. ${ }^{8}$

\section{Conclusion}

The outcome of purely cystic PPB (types I and Ir) is better than the outcomes of more aggressive types. ${ }^{5}$

The rarity and subtle malignant features of type I PPB contribute to difficulty in making the correct diagnosis. Adjuvant chemotherapy appears to benefit patients with type I PPB. A rigorous surveillance schedule after type I
PPB diagnosis might detect early recurrence and be an acceptable alternative to adjuvant chemotherapy if the parents do not favor it. ${ }^{3}$

\section{References}

1 Priest JR, McDermott MB, Bhatia S, Watterson J, Manivel JC, Dehner LP. Pleuropulmonary blastoma: a clinicopathologic study of 50 cases. Cancer 1997;80(01):147-161

2 Manivel JC, Priest JR, Watterson J, et al. Pleuropulmonary blastoma. The so-called pulmonary blastoma of childhood. Cancer 1988;62(08):1516-1526

3 Priest JR, Hill DA, Williams GM, et al; International Pleuropulmonary Blastoma Registry. Type I pleuropulmonary blastoma: a report from the International Pleuropulmonary Blastoma Registry. J Clin Oncol 2006;24(27):4492-4498

4 Priest JR, Williams GM, Hill DA, Dehner LP, Jaffé A. Pulmonary cysts in early childhood and the risk of malignancy. Pediatr Pulmonol 2009;44(01):14-30

5 MacSweeney F, Papagiannopoulos K, Goldstraw P, Sheppard MN, Corrin B, Nicholson AG. An assessment of the expanded classification of congenital cystic adenomatoid malformations and their relationship to malignant transformation. Am J Surg Pathol 2003; 27(08):1139-1146 
e14 PPB Mimicking Congenital Pulmonary Airway Malformation Haider et al.

6 Ch'In KY, Tang MY. Congenital adenomatoid malformation of one lobe of a lung with general anasarca. Arch Pathol (Chic) 1949; 48(03):221-229

7 Stocker JT. Cystic lung disease in infants and children. Fetal Pediatr Pathol 2009;28(04):155-184

8 Messinger YH, Stewart DR, Priest JR, et al. Pleuropulmonary blastoma: a report on 350 central pathology-confirmed pleuropulmonary blastoma cases by the International Pleuropulmonary Blastoma Registry. Cancer 2015;121(02):276-285
9 Feinberg A, Hall NJ, Williams GM, et al. Can congenital pulmonary airway malformation be distinguished from Type I pleuropulmonary blastoma based on clinical and radiological features? J Pediatr Surg 2016;51(01):33-37

10 Oliveira C, Himidan S, Pastor AC, et al. Discriminating preoperative features of pleuropulmonary blastomas (PPB) from congenital cystic adenomatoid malformations (CCAM): a retrospective, age-matched study. Eur J Pediatr Surg 2011; 21(01):2-7 